\title{
A time-resolved Multifocal Multiphoton Microscope for High Speed FRET imaging In Vivo
}

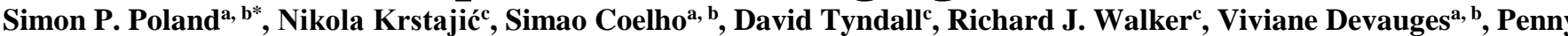
E. Morton ${ }^{\mathrm{b}}$, Nicole S. Nicholas ${ }^{\mathrm{a}}$, Justin Richardson ${ }^{\mathrm{c}}$, David Day-Uei Li ${ }^{\mathrm{d}}$, Klaus Suhling ${ }^{\mathrm{e}}$, Claire M. Wells ${ }^{\mathrm{a}}$, Maddy Parsons $^{\mathrm{b}}$, Robert K. Henderson ${ }^{\mathrm{c}}$ and Simon M. Ameer-Beg ${ }^{\mathrm{a}, \mathrm{b}}$

${ }^{a}$ Division of Cancer Studies, Guy's Campus, Kings College, London, UK; ${ }^{b}$ Randall Division of Cell and Molecular Biophysics, Guy's Campus, Kings College, London, UK ${ }^{c}$ Institute for Integrated Micro and Nano Systems, School of Engineering, University of Edinburgh, Edinburgh, UK; ${ }^{d}$ Centre for Biophotonics, Strathclyde Institute of Pharmacy \& Biomedical Sciences, University of Strathclyde, Glasgow, UK G4 ORE; ${ }^{e}$ Department of Physics, King's College London, Strand, London, UK;

*Corresponding author: simon.poland@kcl.ac.uk

Received Month X, XXXX; revised Month X, XXXX; accepted Month X, XXXX; posted Month X, XXXX (Doc. ID XXXXX); published Month X, XXXX

Imaging the spatio-temporal interaction of proteins in vivo is essential to understanding the complexities of biological systems. The highest accuracy monitoring of protein-protein interactions is achieved using FRET measured by fluorescence lifetime imaging, with measurements taking minutes to acquire a single frame, limiting their use in dynamic live cell systems. We present a diffraction limited, massively parallel, time-resolved multifocal multiphoton microscope capable of producing fluorescence lifetime images with $55 \mathrm{ps}$ time-resolution, giving improvements in acquisition speed of a factor of 64. We present demonstrations with FRET imaging in a model cell system and demonstrate in vivo FLIM using a GTPase biosensor in the zebrafish embryo.

(C) 2014 Optical Society of America

OCIS Codes: (180.0180) Microscopy, (170.2520) Fluorescence Microscopy, (180.4315) Nonlinear microscopy, (040.1240) Detector Arrays, (090.1970) Diffractive Optics

http://dx.doi.org/10.1364/OL.99.099999

Fluorescence lifetime imaging microscopy (FLIM) is a well-established method for high resolution imaging of the functional spatio-temporal dynamics in situ using a variety of techniques, Förster resonance energy transfer (FRET) being by far the most extensively studied for protein-protein homoand hetero-dimer interactions [1, 2]. Multiphoton microscopy confers additional advantages in terms of inherent three dimensional sectioning and enhanced depth penetration for in vivo imaging [3-6]. However, the data acquisition rate for FLIM is a significant limitation in current implementations of laser scanning microscopy.

Time-correlated single photon counting (TCSPC) is considered the gold standard for high precision multiphoton fluorescence lifetime imaging, due to its measurement accuracy [7]. The fundamental limitation associated with TCSPC is imaging speed, due to the stochastic nature of the acquisition process. This limits the counting rate to much less than one photon per excitation event (typically $1 \%$ ) to prevent inaccuracies in lifetime determination due to pulse pile up[8]. For a typical laser scanning FLIM acquisition times are of the order of minutes, whilst the timescales associated with many dynamic biological events are much faster [9]. Whilst resonant scanners can achieve such rates for laser scanning microscopy, often the signal-to-noise is limiting due to both phototoxicity and detection efficiency. One solution is to use a combination of parallelized laser beams with detector arrays (time-gated camera systems [10] or photodiode arrays [11]) to improve the rate of acquisition. Accurate determination of fluorescence lifetime with large numbers of channels in such a parallel manner represents a significant challenge when imaging such complex biological interactions at high temporal resolution.

In this paper, we present a multifocal multiphoton fluorescence lifetime imaging microscope (MMFLIM) which significantly improves the rate of acquisition of high resolution FLIM through the parallelization of both the excitation and detection process. The system is composed of a femtosecond pulsed 2D beamlet array, optically conjugated with a camera consisting of individual single-photon avalanche photodiodes (SPADs), each of which has a quantum efficiency off $28 \%$ at $500 \mathrm{~nm}$ and operates in time-correlated single photon counting mode (Megaframe) [12]. Essentially, the MM-FLIM is composed of 64 separate multiphoton FLIM microscopes working in a parallelized format to facilitate high data acquisition speeds. The Megaframe camera can transfer time-resolved data from the $32 \times 32$ pixel array at 500 kilocounts/sec/pixel to an FPGA for processing. Due to limitations with USB2.0 interface bus data transfer rates, we restrict acquisition to 64 detectors for a maximum data acquisition rate of $16 \mathrm{Mcts} / \mathrm{sec}$. At present this is still 20 times faster than a typical TCSPC single detector setup, where the data acquisition rate is restricted to $800 \mathrm{kcts} / \mathrm{sec}$ ( $1 \%$ of laser repetition rate, $\sim 80 \mathrm{MHz}$ ).

To generate the required multibeam excitation, we developed a phase-domain spatial light modulator based, arbitrary pattern holographic projection system $[10,13,14]$. The beam array was generated by illuminating a pre-calculated phase pattern and conjugated with the back aperture of a microscope 
objective via a scanning system. The integrated microscopy system is shown in schematic in Figure 1. Light from the Chameleon Ultra II Ti:Sapphire laser system (Coherent Inc, CA) operating at $900 \mathrm{~nm}$ was projected onto the Spatial Light Modulator (Boulder Nonlinear Systems Inc, CO) that generates the appropriate beamlet array pattern with a diffraction efficiency of $49 \%$, which was then re-imaged and scanned across sample and using a set of galvanometer mirrors. Two photon generated fluorescence collected by the objective lens was then de-scanned and projected onto the Megaframe SPAD array for detection. Illumination, sample and detection planes were all critically aligned conjugate such that magnification between planes was optimal for both imaging resolution and detection efficiency.

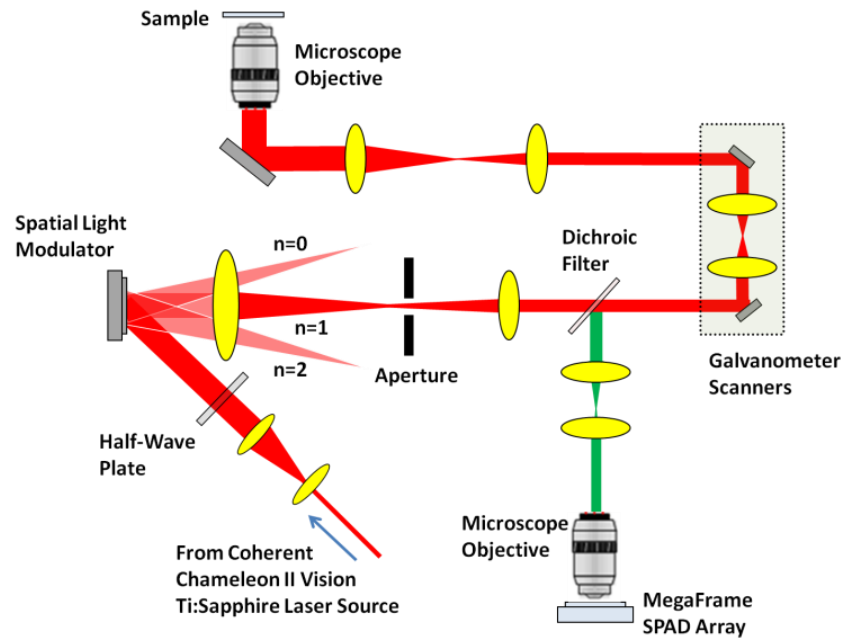

Fig. 1: Schematic of the multifocal multiphoton system

For parallelized TCSPC detection, each fluorescence image of the beamlet array was focused onto a single SPAD of the camera [15]. To ensure optimal efficiency, each fluorescent beamlet generated at the sample plane was projected such that it under-filled the active area $(6 \mu \mathrm{m}$ diameter $)$ of the SPAD. This was achieved by matching the numerical aperture of the illumination and detection such that the diffraction limited image of the fluorescent beamlet has a FHWM of $1.8 \mu \mathrm{m}$. By reimaging the beamlets onto the detector, the optical fill factor was amplified from $1 \%$ to $100 \%$ which provides up to a 10 times improvement over lenslet light collection approaches with similar camera devices [16].

The field of view of the microscope was determined by the magnification between object and detection planes, with beamlets separated in the detection plane by the detector spacing (where each pixel has a pitch of $50 \mu \mathrm{m}$ ). For a 40x imaging objective (Nikon Plan Fluor Oil Immersion 1.3 NA) the field of view for an $8 \times 8$ beamlet array was $100 \mu \mathrm{m}$. Careful alignment of the beamlet spacing onto the detector array was performed. Poor intensity fidelity between beams generated via phase holograms is well-known. To compensate for this and the variable detection efficiency of each individual SPAD (which has a 9\% variability in detection efficiency for the chosen $8 x 8$ array) we implemented a novel doubly weighted Gerchberg-Saxton algorithm (integrating direct signal feedback from the SPAD array)[17], which led to $\sim 7$ times improvement in uniformity of the beam intensity variation across the array.

The microscope system, including $x^{-} y$ scanner control and synchronization with the Megaframe camera was controlled using software written in LabVIEW. For each individual image acquisition, the system processed $32 \times 32$ data points for $8 \times 8$ detectors producing $256 \times 256$ pixel images. The system could be operated in either single plane acquisition mode or a single plane at multiple depths mode to generate a 3 dimensional z-stack.

Imaging sub-diffraction limited $100 \mathrm{~nm}$ dia. fluorescent beads (Life Technologies Ltd, UK), confirmed diffraction limited performance in lateral and axial resolutions of $0.43 \pm 0.03 \mu \mathrm{m}$ and $0.97 \pm 0.04 \mu \mathrm{m}$ respectively for a system with the effective N.A. of 1.2 (Figure 2).

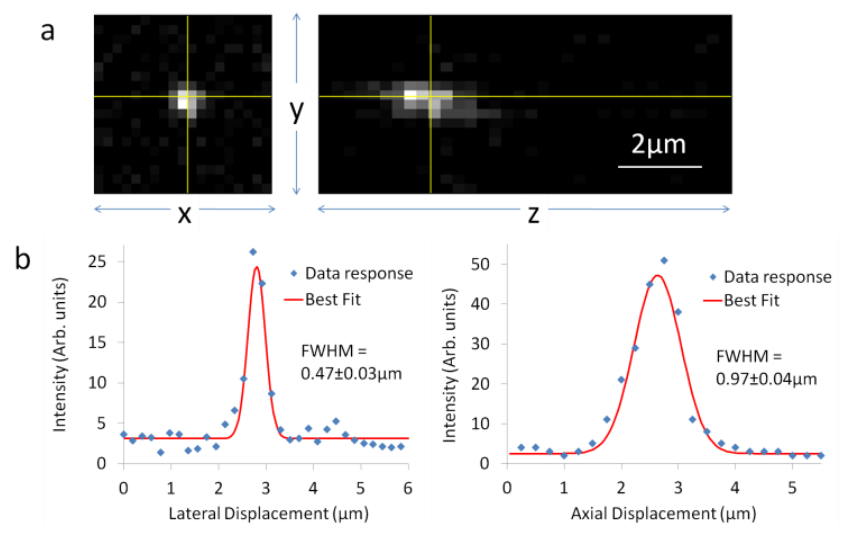

Figure 2: (a) Lateral (XY) and axial (YZ) optical sections of a $0.1 \mu \mathrm{m}$ diameter multispectral sub-diffraction limited fluorescent bead reconstructed from a 3D image stack. (b) Point spread functions taken of the bead to determine the resolutions of the system.

To evaluate the lifetime imaging performance and capabilities of the MM-FLIM imaging system, FRET standards [18] comprising of EGFP and mRFP1 separated with various length amino acid linker sequences were expressed in MCF7 cells, and imaged using the system (Figure 3). Each individual image was acquired for 10 seconds. The FRET efficiencies (as calculated from equation $E=1-\tau / \tau_{\text {Donor }}$, where $\tau$ is the fluorescence lifetime of the probe in the presence of a acceptor and $\tau_{\text {Donor }}$ the unmodified donor lifetime[19]) were shown to increase as the linker size decreases, with data comparable to conventional single beam TCSPC (Table 1). The conventional TCSPC system contained a bialkali photomultiplier tube (Hamamatsu R7401-P) detector with custom built amplifier had a similar quantum efficiency to the MM-FLIM system. The small variability FRET efficiencies between TCSPC and 
MM-FLIM acquired datasets can be accounted for by differences in cell biochemistry between samples. All lifetime data were analysed using $5 \times 5$ circular binning with Levenberg-Marquardt fitting in TRI2 lifetime analysis software.

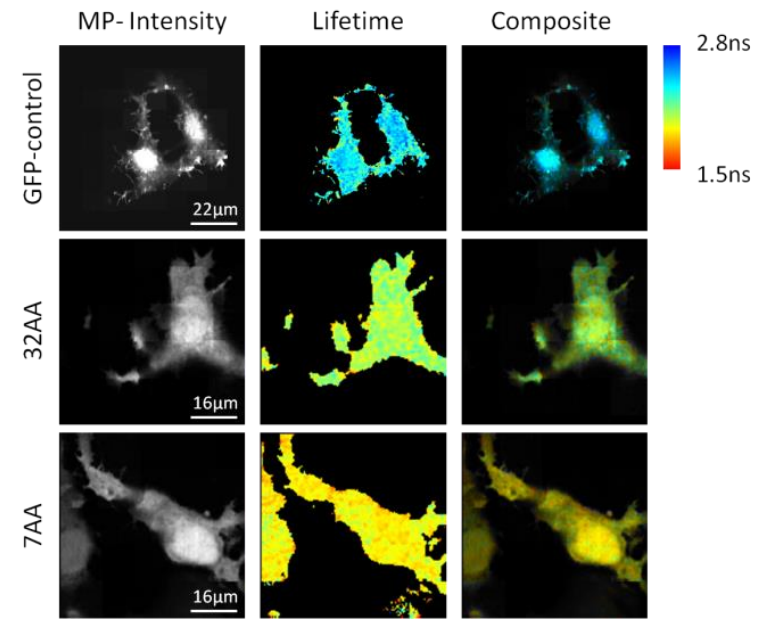

Figure 3: Intensity, lifetime and composite images of MCF7 cells expressing EGFP connected to mRFP using amino acid chains of various sizes compared with MCF7 cells expressing EGFP alone.

Table 1: Comparing FRET efficiencies and associated standard errors of the FRET standards using the MMFLIM imaging system and a conventional single beam TCSPC system.

\begin{tabular}{cccc} 
Transfection & GFP_32aa_RFP & GFP_19aa_RFP & GFP_7aa_RFP \\
\hline $\begin{array}{c}\text { FRET efficiency } \\
\text { conventional } \\
\text { single beam }\end{array}$ & $0.163 \pm 0.003$ & $0.199 \pm 0.001$ & $0.244 \pm 0.003$ \\
TCSPC & $\mathrm{N}=10$ & $\mathrm{~N}=10$ & $\mathrm{~N}=10$ \\
FRET efficiency & $0.154 \pm 0.002$ & $0.184 \pm 0.002$ & $0.224 \pm 0.005$ \\
MM-FLIM & $\mathrm{N}=3$ & $\mathrm{~N}=3$ & $\mathrm{~N}=3$ \\
TCSPC & & & \\
\hline
\end{tabular}

To demonstrate sensitivity and optical sectioning capabilities of the system, we performed analysis of A375M human melanoma cells expressing a recently developed mTFP/VenusRhoA GTPase FRET biosensor [20] injected into zebrafish embryos and allowed to form a tumour mass over 3 days. Embryos were incubated at $28^{\circ} \mathrm{C}$ and $200 \mu \mathrm{M}$ PTU (Phenylthiocarbamide) was added from one days post fertilisation onwards. 2 day post fertilisation, embryos were anaesthetised in $0.5 \mathrm{mM}$ tricaine and A-375MM cells expressing the modified RhoA biosensor were injected into the heart cavity. Embryos were left for $1 \mathrm{hr}$ at $28^{\circ} \mathrm{C}$ to recover, then incubated at $34^{\circ} \mathrm{C}$. 4 days post injection embryos were embedded in $1.5 \%$ low melting point agarose (containing $0.5 \mathrm{mM}$ tricaine), submerged in $25 \mathrm{mM}$ tricaine and imaged immediately. A 40 image series of optical sections in successive planes was acquired in $400 \mathrm{~s}$ (1 section/10s), a time comparable to that required to acquire a single $\mathrm{z}$-section using conventional TCSPC (Figure 4). Whilst, FRET microscopy has been applied to zebrafish embryos in a small number of studies [20,21], this is the first time either RhoA activity or utilization of a xenograft approach have been addressed. Furthermore, 3D FRET projections of cellular masses within a zebrafish embryo have never previously been achieved. Differentials in RhoA activity can be clearly detected in individual cells within the xenograft, where higher levels of RhoA activity predominate at the cell periphery. This is consistent with previous reports [22]. Moreover, the 3D projection enables us to interrogate the architecture of the cell conglomerate and has the potential to provide unprecedented spatio-temporal resolution regarding RhoA activity within cells relative to their $3 \mathrm{D}$ position.

Figure 4: Multiphoton Intensity, Lifetime and combined

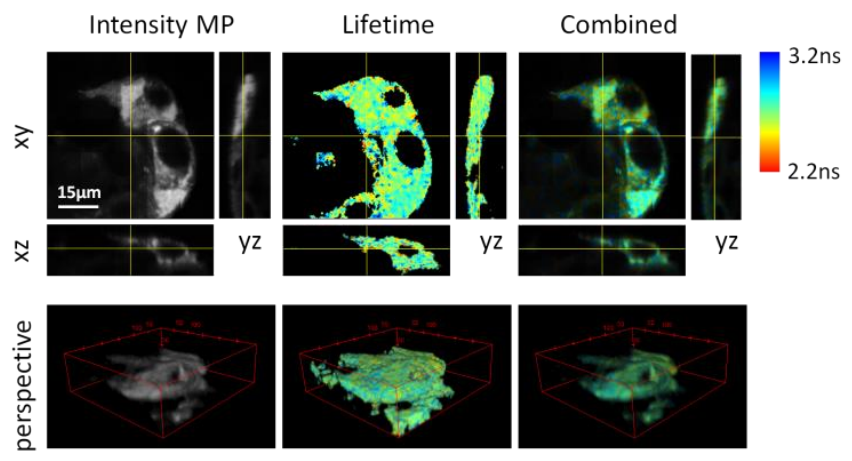

representations of the $3 \mathrm{D}$ data stack heart cavity of a zebrafish 4 days post injection of A-375MM cells expressing the modified RhoA biosensor. (Image size $62.5 \times 62.5 \times 40 \mu \mathrm{m})$.

In summary, we have developed a multifocal multiphoton imaging system for high-frame rate FLIM imaging. We have demonstrated that multifocal multiphoton imaging with a 2D SPAD array is practical, fast and offers novel advantages for fluorescence lifetime imaging. Using beamlet generation and reimaging approach and utilizing small area SPADs ensures optimal efficiency and minimum cross-talk in the system. Moreover, the set-up provides a platform for future improvements in speed and signal-to-noise by increasing the number of beams or using smaller area SPADs. Such advances have the potential to transform timeresolved multiphoton imaging applications in a range of biological systems.

The authors would like to thank: The Biotechnology and Biological Sciences Research Council UK (BB/I022074/1 and BB/I022937/1); STMicroelectronics (Imaging Division, Edinburgh, UK); Medical Research Council UK (MR/K015664/1); Dimbleby Cancer Care endowment fund to King's College London. The Megaframe project has been supported by the European 
Community within the Sixth Framework Programme IST FET Open, http://www.megaframe.eu.

\section{References}

1. J. R. Morris, C. Boutell, M. Keppler, R. Densham, D. Weekes, A. Alamshah, L. Butler, Y. Galanty, L. Pangon, T. Kiuchi, T. Ng, and E. Solomon, "The SUMO modification pathway is involved in the BRCA1 response to genotoxic stress," Nature 462, 886-U877 (2009).

$2 . \quad$ M. Peter, S. M. Ameer-Beg, M. K. Hughes, M. D. Keppler, S. Prag, M. Marsh, B. Vojnovic, and T. $\mathrm{Ng}$, "Multiphoton-FLIM quantification of the EGFPmRFP1 FRET pair for localization of membrane receptor-kinase interactions," Biophys J 88, 12241237 (2005).

3. W. R. Zipfel, R. M. Williams, and W. W. Webb, "Nonlinear magic: multiphoton microscopy in the biosciences," Nat Biotechnol 21, 1368-1376 (2003).

4. E. B. Brown, R. B. Campbell, Y. Tsuzuki, L. $\mathrm{Xu}$, P. Carmeliet, D. Fukumura, and R. K. Jain, "In vivo measurement of gene expression, angiogenesis and physiological function in tumors using multiphoton laser scanning microscopy (vol 7, pg 864, 2001)," Nat Med 7, 1069-1069 (2001).

$5 . \quad$ W. Denk, J. H. Strickler, and W. W. Webb, "2-Photon Laser Scanning Fluorescence Microscopy," Science 248, 73-76 (1990).

6. F. Helmchen, and W. Denk, "Deep tissue twophoton microscopy," Nat Methods 2, 932-940 (2005).

7. E. Gratton, S. Breusegem, J. Sutin, Q. Ruan, and N. Barry, "Fluorescence lifetime imaging for the two-photon microscope: time-domain and frequencydomain methods," J Biomed Opt 8, 381-390 (2003).

8. K. Suhling, D. McLoskey, and D. Birch, "Multiplexed single-photon counting. II. The statistical theory of time-correlated measurements," Rev Sci Instrum 67, 2238-2246 (1996).

9. A. Miyawaki, O. Griesbeck, R. Heim, and R. Y. Tsien, "Dynamic and quantitative $\mathrm{Ca} 2+$ measurements using improved cameleons," Proceedings of the National Academy of Sciences 96, 2135-2140 (1999).

10. S. Padilla-Parra, N. Auduge, M. CoppeyMoisan, and M. Tramier, "Quantitative FRET analysis by fast acquisition time domain FLIM at high spatial resolution in living cells," Biophysical Journal 95, 2976-2988 (2008).

11. S. Kumar, C. Dunsby, P. A. A. De Beule, D. M. Owen, U. Anand, P. M. P. Lanigan, R. K. P. Benninger, D. M. Davis, M. A. A. Neil, P. Anand, C. Benham, A. Naylor, and P. M. W. French, "Multifocal multiphoton excitation and time correlated single photon counting detection for 3-D fluorescence lifetime imaging," Optics Express 15, 12548-12561 (2007).

12. J. Richardson, R. Walker, L. Grant, D. Stoppa, F. Borghetti, E. Charbon, M. Gersbach, and R. K. Henderson, "A 32× 32 50ps resolution 10 bit time to digital converter array in 130nm CMOS for time correlated imaging," in Custom Integrated
Circuits Conference, 2009. CICC'09. IEEE(IEEE2009), pp. 77-80.

13. J. Leach, K. Wulff, G. Sinclair, P. Jordan, J. Courtial, L. Thomson, G. Gibson, K. Karunwi, J. Cooper, Z. J. Laczik, and M. Padgett, "Interactive approach to optical tweezers control," Appl Optics 45, 897-903 (2006).

14. R. A. Colyer, G. Scalia, I. Rech, A. Gulinatti, M. Ghioni, S. Cova, S. Weiss, and X. Michalet, "Highthroughput FCS using an LCOS spatial light modulator and an 8× 1 SPAD array," Biomedical optics express 1, 1408-1431 (2010).

15. J. Richardson, R. Walker, L. Grant, D. Stoppa, F. Borghetti, E. Charbon, M. Gersbach, and R. K. Henderson, "A 32x32 50ps Resolution 10 bit Time to Digital Converter Array in 130nm CMOS for Time Correlated Imaging," Ieee Cust Integr Cir, 7780 (2009).

16. J. M. Pavia, M. Wolf, and E. Charbon, "Measurement and modeling of microlenses fabricated on single-photon avalanche diode arrays for fill factor recovery," Optics express 22, 4202-4213 (2014).

17. S. P. Poland, N. Krstajić, R. D. Knight, R. K. Henderson, and S. M. Ameer-Beg, "Development of a doubly weighted Gerchberg-Saxton algorithm for use in multibeam imaging applications," Optics Letters 39, 2431-2434 (2014).

18. D. R. Matthews, G. O. Fruhwirth, G. Weitsman, L. M. Carlin, E. Ofo, M. Keppler, P. R. Barber, I. D. Tullis, B. Vojnovic, and T. Ng, "A multifunctional imaging approach to high-content protein interaction screening," PloS one 7, e33231 (2012).

19. E. A. Jares-Erijman, and T. M. Jovin, "FRET imaging," Nat Biotechnol 21, 1387-1395 (2003).

20. R. D. Fritz, M. Letzelter, A. Reimann, K. Martin, L. Fusco, L. Ritsma, B. Ponsioen, E. Fluri, S. Schulte-Merker, J. van Rheenen, and O. Pertz, "A Versatile Toolkit to Produce Sensitive FRET Biosensors to Visualize Signaling in Time and Space," Science Signaling 6 (2013).

21. H. Xu, E. Kardash, S. Chen, E. Raz, and F. Lin, "Gbetagamma signaling controls the polarization of zebrafish primordial germ cells by regulating Rac activity," Development 139, 57-62 (2012).

22. P. Nalbant, Y. C. Chang, J. Birkenfeld, Z. F. Chang, and G. M. Bokoch, "Guanine nucleotide exchange factor-H1 regulates cell migration via localized activation of RhoA at the leading edge," Mol Biol Cell 20, 4070-4082 (2009). 